\title{
Crystals from categorified quantum groups
}

\author{
Aaron D. Lauda 1 \#and Monica Vazirani»" \\ ${ }^{1}$ Department of Mathematics, Columbia University, New York, NY 10027 \\ ${ }^{2}$ Department of Mathematics, University of California, Davis, Davis, California 95616-8633
}

\begin{abstract}
We study the crystal structure on categories of graded modules over algebras which categorify the negative half of the quantum Kac-Moody algebra associated to a symmetrizable Cartan data. We identify this crystal with Kashiwara's crystal for the corresponding negative half of the quantum Kac-Moody algebra. As a consequence, we show the simple graded modules for certain cyclotomic quotients carry the structure of highest weight crystals, and hence compute the rank of the corresponding Grothendieck group.

Résumé. Nous étudions la structure cristalline sur les catégories de modules gradués sur algèbres qui categorify la moitié négative du quantum de Kac-Moody algèbre associée à un ensemble de data symétrisables Cartan. Nous identifions ce cristal avec des cristaux de Kashiwara pour le négatif correspondant la moitié de l'algèbre de Kac-Moody quantum. En conséquence, nous montrons la simples modules classés pour certains quotients cyclotomique porter le structure des cristaux de poids le plus élevé, et donc de calculer le rang de le groupe correspondant Grothendieck.
\end{abstract}

Keywords: Khovanov-Lauda-Rouquier algebras, quiver Hecke algebras, categorification

\section{Introduction}

In [KL09, KL08a] a family $R$ of graded algebras was introduced that categorifies the integral form ${ }_{\mathcal{A}} \mathbf{U}_{q}^{-}:={ }_{\mathcal{A}} \mathbf{U}_{q}^{-}(\mathfrak{g})$ of the negative half of the quantum enveloping algebra $\mathbf{U}_{q}(\mathfrak{g})$ associated to a symmetrizable Kac-Moody algebra $\mathfrak{g}$. Similar algebras were also independently introduced by Rouquier [Rou08]. The grading on these algebras equips the Grothendieck group $K_{0}(R-$ pmod) of the category of finitely-generated graded projective $R$-modules with the structure of a $\mathbb{Z}\left[q, q^{-1}\right]$-module. Natural parabolic induction and restriction functors give $K_{0}\left(R\right.$-pmod) the structure of a (twisted) $\mathbb{Z}\left[q, q^{-1}\right]$ bialgebra. In [KL09, KL08a] an explicit isomorphism of twisted bialgebras was given between ${ }_{\mathcal{A}} \mathbf{U}_{q}^{-}$and $K_{0}(R-$ pmod $)$.

Several conjectures were also made in [KL09, KL08a]. One conjecture that remains unsolved is the so called cyclotomic quotient conjecture which suggests a close connection between certain finite dimensional quotients of the algebras $R$ and the integrable representation theory of quantum Kac-Moody algebras. While this conjecture has been proven in finite and affine type $A$ by Brundan and Kleshchev [BK09], very little is known in the case of an arbitrary symmetrizable Cartan datum. Here we show that simple graded modules for these cyclotomic quotients carry the structure of highest weight crystals. Hence we

\footnotetext{
${ }^{\dagger}$ partially supported by the NSF grants DMS-0739392 and DMS-0855713

${ }^{\ddagger}$ partially supported by the NSA grant \#H982300910076 
identify the rank of the corresponding Grothendieck group with the rank of the integral highest weight representation, thereby laying to rest a major component of the cyclotomic conjecture.

This submission is an extended abstract of the preprint [LV09]. We refer the reader there the complete proofs and details, as well as more background and context on these results.

\section{The algebra $R(\nu)$}

We are given a Cartan data: the weight lattice $P$, simple roots $\alpha_{i}$ indexed by $i \in I$, simple coroots $h_{i} \in P^{\vee}=\operatorname{Hom}_{\mathbb{Z}}(P, \mathbb{Z})$, a bilinear form $():, P \times P \rightarrow \mathbb{Z}$, and the canonical pairing $\langle\cdot, \cdot\rangle: P^{\vee} \times P \rightarrow \mathbb{Z}$, such that $\left[\left\langle h_{i}, \alpha_{j}\right\rangle\right]_{i, j \in I}$ is a symmetrizable generalized Cartan matrix. In what follows we write $a_{i j}=$ $-\langle i, j\rangle:=-\left\langle h_{i}, \alpha_{j}\right\rangle$ for $i, j \in I$. Let $\Lambda_{i} \in P^{+}$be the fundamental weights defined by $\left\langle h_{j}, \Lambda_{i}\right\rangle=\delta_{i j}$. Let $q_{i}=q^{\frac{\left(\alpha_{i}, \alpha_{i}\right)}{2}},[a]_{i}=\frac{q_{i}^{a}-q_{i}^{-a}}{q_{i}-q_{i}^{-1}},[a]_{i} !=[a]_{i}[a-1]_{i} \ldots[1]_{i}$.

Recall the definition from [KL09, KL08a] of the algebra $R$ associated to a Cartan datum. Let $\mathbb{k}$ be an algebraically closed field (of arbitrary characteristic).

For $\nu=\sum_{i \in I} \nu_{i} \cdot i \in \mathbb{N}[I]$ let $\operatorname{Seq}(\nu)$ be the set of all sequences of vertices $\boldsymbol{i}=i_{1} \ldots i_{m}$ where $i_{r} \in I$ for each $r$ and vertex $i$ appears $\nu_{i}$ times in the sequence. The length $m$ of the sequence is equal to $|\nu|=\sum_{i \in I} \nu_{i}$. It is sometimes convenient to identify $\nu=\sum_{i \in I} \nu_{i} \cdot i \in \mathbb{N}[I]$ as $\nu \in \sum_{i \in I} \nu_{i} \alpha_{i} \in Q_{+}=$ $\oplus_{i \in I} \mathbb{Z}_{\geq 0} \alpha_{i}$.

For $\nu \in \mathbb{N}[I]$ with $|\nu|=m$, let $R(\nu)$ denote the associative, $\mathbb{k}$-algebra on generators $1_{i}$ for $\boldsymbol{i} \in \operatorname{Seq}(\nu)$, $x_{r}$ for $1 \leq r \leq m$, and $\psi_{r}$ for $1 \leq r \leq m-1$ subject to the following relations for $\boldsymbol{i}, \boldsymbol{j} \in \operatorname{Seq}(\nu)$ :

$$
\begin{aligned}
& 1_{i} 1_{j}=\delta_{i, j} 1_{i}, \quad x_{r} 1_{i}=1_{i} x_{r}, \quad \psi_{r} 1_{i}=1_{s_{r}(i)} \psi_{r}, \\
& x_{r} x_{t}=x_{t} x_{r}, \quad \psi_{r} \psi_{t}=\psi_{t} \psi_{r} \quad \text { if }|r-t|>1 \text {, } \\
& \psi_{r} \psi_{r} 1_{i}= \begin{cases}0 & \text { if } i_{r}=i_{r+1} \\
1_{i} & \text { if }\left(\alpha_{i_{r}}, \alpha_{i_{r+1}}\right)=0 \\
\left(x_{r}^{-\left\langle i_{r}, i_{r+1}\right\rangle}+x_{r+1}^{-\left\langle i_{r+1}, i_{r}\right\rangle}\right) 1_{i} & \text { if }\left(\alpha_{i_{r}}, \alpha_{i_{r+1}}\right) \neq 0 \text { and } i_{r} \neq i_{r+1},\end{cases} \\
& \left(\psi_{r} \psi_{r+1} \psi_{r}-\psi_{r+1} \psi_{r} \psi_{r+1}\right) 1_{i}= \\
& = \begin{cases}\sum_{t=0}^{-\left\langle i_{r}, i_{r+1}\right\rangle-1} x_{r}^{t} x_{r+2}^{-\left\langle i_{r}, i_{r+1}\right\rangle-1-t} 1_{i} & \text { if } i_{r}=i_{r+2} \text { and }\left(\alpha_{i_{r}}, \alpha_{i_{r+1}}\right) \neq 0 \\
0 & \text { otherwise, }\end{cases} \\
& \left(\psi_{r} x_{t}-x_{s_{r}(t)} \psi_{r}\right) 1_{i}=\left\{\begin{array}{cl}
1_{i} & \text { if } t=r \text { and } i_{r}=i_{r+1} \\
-1_{i} & \text { if } t=r+1 \text { and } i_{r}=i_{r+1} \\
0 & \text { otherwise }
\end{array}\right.
\end{aligned}
$$

The algebra is graded with generators defined to have degrees $\operatorname{deg}\left(1_{i}\right)=0, \operatorname{deg}\left(x_{r} 1_{i}\right)=\left(\alpha_{i_{r}}, \alpha_{i_{r}}\right)$, and $\operatorname{deg}\left(\psi_{r} 1_{i}\right)=-\left(\alpha_{i_{r}}, \alpha_{i_{r+1}}\right)$.

We let the (nonunital) algebra $R$ be defined by $R=\bigoplus_{\nu \in \mathbb{N}[I]} R(\nu)$.

Rouquier has independently defined a generalization of the algebras $R$, where the relations depend on Hermitian matrices [Rou08]. 
Remark 2.1 For $\boldsymbol{i}, \boldsymbol{j} \in \operatorname{Seq}(\nu)$ let $_{j} S_{\boldsymbol{i}}$ be the subset of $S_{m}$ consisting of permutations $w$ that take $\boldsymbol{i}$ to $\boldsymbol{j}$ via the standard action of permutations on sequences. Denote the subset $\{\widehat{w}\}_{w \in \in_{j} S_{i}}$ of $1_{j} R 1_{i}$ by ${ }_{j} \widehat{S}_{i}$. It was shown in [KL09 KL08a] that the vector space $1_{j} R(\nu) 1_{i}$ has a basis consisting of elements of the form $\left\{\psi_{\widehat{w}} \cdot x_{1}^{a_{1}} \cdots x_{m}^{a_{m}} 1_{i} \mid \widehat{w} \in \widehat{S}_{j}, \quad a_{r} \in \mathbb{Z}_{\geq 0}\right\}$.

Let $w_{0}$ denote the longest element of $S_{|\nu|}$. We define an involution $\sigma=\sigma_{\nu}: R(\nu) \rightarrow R(\nu)$ by $\sigma\left(1_{i}\right)=1_{w_{0}(i)}, \sigma\left(x_{r}\right)=x_{|\nu|+1-r}, \sigma\left(\psi_{r}\right)=\psi_{|\nu|-r}$. Given an $R(\nu)$-module $M$, we let $\sigma^{*} M$ denote the $R(\nu)$-module whose underlying set is $M$ but with twisted action $r \cdot u=\sigma(r) u$.

Define the character $\operatorname{ch}(M)$ of an $R(\nu)$-module $M$ as $\operatorname{ch}(M)=\sum_{i \in \operatorname{Seq}(\nu)} \operatorname{gdim}\left(1_{i} M\right) \cdot \boldsymbol{i}$, where gdim denotes the graded dimension. When $M$ is finite dimensional, $\operatorname{ch}(M)$ is an element of the free $\mathbb{Z}\left[q, q^{-1}\right]$-module with basis $\operatorname{Seq}(\nu)$.

\section{Functors on the modular category}

Let $R(\nu)$-fmod be the category of finite dimensional graded $R(\nu)$-modules. The morphisms are grading-preserving module homomorphisms. Note that this category contains all of the simples. Henceforth, by an $R(\nu)$-module we will mean a finite dimensional graded $R(\nu)$-module, unless we say otherwise. We will denote the zero module by $\mathbf{0}$. Let $R-\mathrm{fmod} \stackrel{\text { def }}{=} \bigoplus_{\nu \in \mathbb{N}[I]} R(\nu)-\mathrm{fmod}$.

For any two $R(\nu)$-modules $M, N$ denote by $\operatorname{Hom}(M, N)$ or $\operatorname{Hom}_{R(\nu)}(M, N)$ the $\mathbb{k}$-vector space of degree preserving homomorphisms, and by $\operatorname{Hom}(M\{r\}, N)=\operatorname{Hom}(M, N\{-r\})$ the space of homogeneous homomorphisms of degree $r$. Here $N\{r\}$ denotes $N$ with the grading shifted up by $r$, so that $\operatorname{ch}(N\{r\})=q^{r} \operatorname{ch}(N)$. Then we write $\operatorname{HOM}(M, N):=\bigoplus_{r \in \mathbb{Z}} \operatorname{Hom}(M, N\{r\})$, for the $\mathbb{Z}$-graded $\mathbb{k}$-vector space of all $R(\nu)$-module morphisms.

Though it is essential to work with the degree preserving morphisms to get the $\mathbb{Z}\left[q, q^{-1}\right]$-module structure for the categorification theorems in [KL09, KL08a], for our purposes it will often be convenient to work with degree homogenous morphisms, but not necessarily degree preserving. Since any homogenous morphism can be interpreted as a degree preserving morphism by shifting the grading on the source or target, all results stated using homogeneous morphisms can be recast as degree zero morphisms for an appropriate shift on the source or target.

\subsection{Induction and Restriction functors}

There is an inclusion of graded algebras

$$
\iota_{\nu, \nu^{\prime}}: R(\nu) \otimes R\left(\nu^{\prime}\right) \hookrightarrow R\left(\nu+\nu^{\prime}\right)
$$

taking the idempotent $1_{i} \otimes 1_{j}$ to $1_{i j}$ and the unit element $1_{\nu} \otimes 1_{\nu^{\prime}}$ to an idempotent of $R\left(\nu+\nu^{\prime}\right)$ denoted $1_{\nu, \nu^{\prime}}$. This inclusion gives rise to restriction and induction functors denoted by $\operatorname{Res}_{\nu, \nu^{\prime}}$ and $\operatorname{Ind}_{\nu, \nu^{\prime}}$, respectively. When it is clear from the context, or when no confusion is likely to arise, we often simplify notation and write Res and Ind.

We can also consider these notions for any tuple $\underline{\nu}=\left(\nu^{(1)}, \nu^{(2)}, \ldots, \nu^{(k)}\right)$ and sometimes refer to the image $R(\underline{\nu}) \stackrel{\text { def }}{=} \operatorname{Im} \iota_{\underline{\nu}} \subseteq R\left(\nu^{(1)}+\cdots+\nu^{(k)}\right)$ as a parabolic subalgebra. This subalgebra has identity $1_{\underline{\nu}}$. Let $\mu=\nu^{(1)}+\cdots+\nu^{(k)}, m=\sum_{r}\left|\nu^{(r)}\right|$, and $P=P_{\underline{\nu}}$ be the composition $\left(\left|\nu^{(1)}\right|, \ldots,\left|\nu^{(k)}\right|\right)$ of $m$ so that $S_{P}$ is the corresponding parabolic subgroup of $S_{m}$. It follows from Remark 2.1 that $R(\mu) 1_{\underline{\nu}}$ is a free right $R(\underline{\nu})$-module with basis $\left\{\psi_{\widehat{w}} 1_{\underline{\nu}} \mid w \in S_{m} / S_{P}\right\}$ and $1_{\underline{\nu}} R(\mu)$ is a free left $R(\underline{\nu})$-module with 
basis $\left\{1_{\underline{\nu}} \psi_{\widehat{w}} \mid w \in S_{P} \backslash S_{m}\right\}$. By abuse of notation we will write $S_{m} / S_{P}$ to denote the minimal length left coset representatives, i.e. $\left\{w \in S_{m} \mid \ell(w v)=\ell(w)+\ell(v) \forall v \in S_{P}\right\}$, and $S_{P} \backslash S_{m}$ for the minimal length right coset representatives.

Remark 3.1 It is easy to see that if $M$ is an $R(\underline{\nu})$-module with basis $\mathcal{U}$ consisting of weight vectors, then $\left\{\psi_{\widehat{w}} \otimes u \mid u \in \mathcal{U}, w \in S_{m} / S_{P}\right\}$ is a weight basis of $\operatorname{Ind}_{\nu} M \stackrel{\text { def }}{=} R(\mu) \otimes_{R(\nu)} M$ (where for each $w$ we fix just one reduced expression $\widehat{w})$. Note $R(\mu) \otimes_{R(\underline{\nu})} M=\bar{R}(\mu) 1_{\underline{\nu}} \otimes_{R(\underline{\nu})} M$ since $\psi_{\widehat{w}} 1_{\underline{\nu}} \otimes u=\psi_{\widehat{w}} \otimes 1_{\underline{\nu}} u=$ $\psi_{\widehat{w}} \otimes u$.

One extremely important property of the functor $\operatorname{Ind}_{\underline{\nu}}-\stackrel{\text { def }}{=} R(\mu) \otimes_{R(\underline{\nu})}-$ is that it is left adjoint to restriction, i.e., there is a functorial isomorphism $\operatorname{HOM}_{R(\mu)}\left(\operatorname{Ind}_{\underline{\nu}} A, B\right) \cong \operatorname{HOM}_{R(\underline{\nu})}\left(A, \operatorname{Res}_{\underline{\underline{\nu}}} B\right)$ where $A, B$ are finite dimensional $R(\underline{\nu})$ - and $R(\mu)$-modules, respectively. We refer to this property as Frobenius reciprocity and use it repeatedly, often for deducing information about characters.

\subsection{Refining the restriction functor}

For $M$ in $R(\nu)-\bmod$ and $i \in I$ let $\Delta_{i} M=\left(1_{\nu-i} \otimes 1_{i}\right) M=\operatorname{Res}_{\nu-i, i} M$, and, more generally, $\Delta_{i^{n}} M=$ $\left(1_{\nu-n i} \otimes 1_{n i}\right) M=\operatorname{Res}_{\nu-n i, n i} M$. We view $\Delta_{i^{n}}$ as a functor into the category $R(\nu-n i) \otimes R(n i)-\bmod$. By Frobenius reciprocity, there are functorial isomorphisms

$$
\operatorname{HOM}_{R(\nu)}\left(\operatorname{Ind}_{\nu-n i, n i} N \otimes L\left(i^{n}\right), M\right) \cong \operatorname{HOM}_{R(\nu-n i) \otimes R(n i)}\left(N \otimes L\left(i^{n}\right), \Delta_{i^{n}} M\right),
$$

for $M$ as above and $N \in R(\nu-n i)-\bmod$.

Define

$$
e_{i}:=\operatorname{Res}_{\nu-i}^{\nu-i, i} \circ \Delta_{i}: R(\nu)-\bmod \rightarrow R(\nu-i)-\bmod
$$

and for $M$ an irreducible $R(\nu)$-module, set

$$
\widetilde{e}_{i} M:=\operatorname{soc} e_{i} M, \quad \widetilde{f}_{i} M:=\operatorname{cosoc} \operatorname{Ind}_{\nu, i}^{\nu+i} M \otimes L(i), \quad \varepsilon_{i}(M):=\max \left\{n \geq 0 \mid \widetilde{e}_{i}^{n} M \neq \mathbf{0}\right\} .
$$

We also define their $\sigma$-symmetric versions, which are indicated with a $\vee$. Note that $\sigma^{*}\left(\Delta_{i}\left(\sigma^{*} M\right)\right)=$ $\operatorname{Res}_{i, \nu-i} M$. Set $e_{i}^{\vee}:=\sigma^{*}\left(e_{i}\left(\sigma^{*} M\right)\right), \widetilde{e}_{i}^{\vee} M:=\sigma^{*}\left(\widetilde{e}_{i}\left(\sigma^{*} M\right)\right), \widetilde{f}_{i}^{\vee} M:=\sigma^{*}\left(\widetilde{f}_{i}\left(\sigma^{*} M\right)\right), \varepsilon_{i}^{\vee}(M):=$ $\varepsilon_{i}\left(\sigma^{*} M\right)$. Observe that the functors $e_{i}$ and $e_{i}^{\vee}$ are exact. It is a theorem of [KL09] that if $M$ is irreducible, so are $\widetilde{f}_{i} M$ and $\widetilde{e_{i}} M$ (so long as the latter is nonzero), and likewise for $\widetilde{f}_{i}^{\vee} M$ and ${\widetilde{e_{i}}}^{\vee} M$. For other key properties of the functors ${\widetilde{e_{i}}}_{i}$ and $\widetilde{f}_{i}$ on simple modules, see [KL09] or [LV09].

\subsection{The cyclotomic algebras $R^{\Lambda}(\nu)$}

For $\Lambda=\sum_{i \in I} \lambda_{i} \Lambda_{i} \in P^{+}$consider the two-sided ideal $\mathcal{J}_{\nu}^{\Lambda}$ of $R(\nu)$ generated by elements $\left(x_{1} 1_{i}\right)^{\lambda_{i_{1}}}$ over all sequences $i \in \operatorname{Seq}(\nu)$. We sometimes write $\mathcal{J}_{\nu}^{\Lambda}=\mathcal{J}^{\Lambda}$ when no confusion is likely to arise. Define

$$
R^{\Lambda}(\nu):=R(\nu) / \mathcal{J}_{\nu}^{\Lambda}
$$

By analogy with the Ariki-Koike cyclotomic quotient of the affine Hecke algebra [AK94] (see also [Ari02]) this algebra is called the cyclotomic quotient at weight $\Lambda$ of $R(\nu)$. As above we form the nonunital ring

$$
R^{\Lambda}=\bigoplus_{\nu \in \mathbb{N}[I]} R^{\Lambda}(\nu)
$$


For bookkeeping purposes we will denote $R^{\Lambda}(\nu)$ modules by $\mathcal{M}$ but $R(\nu)$-modules by $M$.

We introduce functors

$$
\operatorname{infl}_{\Lambda}: R^{\Lambda}(\nu)-\bmod \rightarrow R(\nu)-\mathrm{fmod} \quad \operatorname{pr}_{\Lambda}: R(\nu)-\mathrm{fmod} \rightarrow R^{\Lambda}(\nu)-\bmod
$$

where $\operatorname{infl}_{\Lambda}$ is the inflation along the epimorphism $R(\nu) \rightarrow R^{\Lambda}(\nu)$, so that $\mathcal{M}=\operatorname{infl} \mathcal{M}_{\Lambda}$ on the level of sets. If $\mathcal{M}, \mathcal{N}$ are $R^{\Lambda}(\nu)$-modules, then $\operatorname{Hom}_{R^{\Lambda}(\nu)}(\mathcal{M}, \mathcal{N}) \cong \operatorname{Hom}_{R(\nu)}\left(\operatorname{infl}_{\Lambda} \mathcal{M}\right.$, infl $\left.\mathcal{N}_{\Lambda}\right)$. Note $\mathcal{M}$ is irreducible if and only if $\operatorname{infl}_{\Lambda} \mathcal{M}$ is. We define $\operatorname{pr}_{\Lambda} M=M / \mathcal{J}^{\Lambda} M$. If $M$ is irreducible then $\operatorname{pr}_{\Lambda} M$ is either irreducible or zero. Observe $\operatorname{infl}_{\Lambda}$ is an exact functor and its left adjoint is $\mathrm{pr}_{\Lambda}$ which is only right exact.

A careful study of the modules $L\left(i^{m}\right)$ yields that for simple modules $M$, the algebraic statement $\mathcal{J}^{\Lambda} M=\mathbf{0}$ is equivalent to the measurement that $\varepsilon_{i}^{\vee}(M) \leq \lambda_{i}$ for all $i \in I$, see [Lau09, Proposition 2.8]. Likewise $\mathcal{J}^{\Lambda} M=M$ if and only if there exists some $i \in I$ such that $\varepsilon_{i}^{\vee}(M)>\lambda_{i}$. Hence, given a finite dimensional $R(\nu)$-module $M$, there exists a $\Lambda \in P^{+}$such that $\mathcal{J}^{\Lambda} M=\mathbf{0}$, so that we can identify $M$ with the $R^{\Lambda}(\nu)$-module $\operatorname{pr}_{\Lambda} M$. For instance, take any $\Lambda=\sum_{i \in I} m_{i} \lambda_{i}$ with $m_{i}>\operatorname{dim}_{\mathbb{k}} M$. We deduce the following remark.

Remark 3.2 Let $M$ be a simple $R(\nu)$-module. Then $\operatorname{pr}_{\Lambda} M \neq 0$ iff $\varepsilon_{i}^{\vee}(M) \leq \lambda_{i}$ for all $i \in I$.

Let $\mathcal{M}$ be an irreducible $R^{\Lambda}(\nu)$-module. As in Section 3.2 define $e_{i}^{\Lambda} \mathcal{M}:=\operatorname{pr}_{\Lambda} \circ e_{i} \circ \operatorname{infl}_{\Lambda} \mathcal{M}$ which is a functor $R^{\Lambda}(\nu)-\bmod \rightarrow R^{\Lambda}(\nu-i)-\bmod$, as well as $\widetilde{e}_{i}^{\Lambda} \mathcal{M}=\operatorname{pr}_{\Lambda} \circ \widetilde{e}_{i} \circ \operatorname{infl}_{\Lambda} \mathcal{M}, \tilde{f}_{i}{ }^{\Lambda} \mathcal{M}=\operatorname{pr}_{\Lambda} \circ \widetilde{f}_{i} \circ$ $\operatorname{infl}_{\Lambda} \mathcal{M}, \varepsilon_{i}^{\Lambda}(\mathcal{M})=\varepsilon_{i}\left(\operatorname{infl}_{\Lambda} \mathcal{M}\right)$. Let $\mathcal{M} \in R^{\Lambda}(\nu)-\bmod$ and $M=\operatorname{infl}_{\Lambda} \mathcal{M}$. Then $\operatorname{pr}_{\Lambda} M=\mathcal{M}$. Since $\mathcal{J}^{\Lambda} M=\mathbf{0}$ then $\mathcal{J}^{\Lambda} e_{i} M=\mathbf{0}$ too, so that $e_{i}^{\Lambda} \mathcal{M}$ is an $R(\nu-i)^{\Lambda}$-module with $\operatorname{infl}_{\Lambda}\left(e_{i}^{\Lambda} \mathcal{M}\right)=e_{i} M$. In particular, $\operatorname{dim}_{\mathbb{k}} e_{i}^{\Lambda} \mathcal{M}=\operatorname{dim}_{\mathbb{k}} e_{i} M$. If furthermore $\mathcal{M}$ is irreducible, then ${\widetilde{e_{i}}}^{\Lambda} \mathcal{M}=\operatorname{soc} e_{i}^{\Lambda} \mathcal{M}$.

\subsection{Operators on the Grothendieck group}

Let the Grothendieck groups $G_{0}(R)=\bigoplus_{\nu \in \mathbb{N}[I]} G_{0}(R(\nu)$-fmod $), G_{0}\left(R^{\Lambda}\right)=\bigoplus_{\nu \in \mathbb{N}[I]} G_{0}\left(R^{\Lambda}(\nu)-\right.$ fmod $)$. They have the structure of a $\mathbb{Z}\left[q, q^{-1}\right]$-module given by shifting the grading, $q[M]=[M\{1\}]$.

The functor $e_{i}$ defined in (1) is clearly exact so descends to an operator on the Grothendieck group $G_{0}(R(\nu)-\bmod ) \longrightarrow G_{0}(R(\nu-i)-\bmod )$ and hence $e_{i}: G_{0}(R) \longrightarrow G_{0}(R)$. By abuse of notation, we will also call this operator $e_{i}$. Likewise $e_{i}^{\Lambda}: G_{0}\left(R^{\Lambda}\right) \longrightarrow G_{0}\left(R^{\Lambda}\right)$. We also define divided powers $e_{i}^{(r)}: G_{0}(R) \longrightarrow G_{0}(R)$ given by $e_{i}^{(r)}[M]=\frac{1}{[r]_{i}^{!}}\left[e_{i}^{r} M\right]$, which are well-defined.

For irreducible $M$, we define $\widetilde{e}_{i}[M]=\left[\widetilde{e}_{i} M\right], \widetilde{f}_{i}[M]=\left[\widetilde{f}_{i} M\right]$, and extend the action linearly.

The quantum Serre relations (2) are certain (minimal) relations that hold among the operators $e_{i}$ on $G_{0}(R)$. The operator

$$
\sum_{r=0}^{a+1}(-1)^{r} e_{i}^{(a+1-r)} e_{j} e_{i}^{(r)}=0
$$

In Section 6.2 below, we give an alternate proof to that of Khovanov-Lauda that the quantum Serre relation (2) holds by examining the structure of all simple $R((a+1) i+j)$-modules. We further construct simple $R(c i+j)$-modules that are witness to the nonvanishing of the analogous relation taking $c \leq a$. 


\section{Reminders on crystals}

A main result of this paper is the realization of a crystal graph structure on $G_{0}(R)$ which we identify as the crystal $B(\infty)$. We assume the reader is familiar with the language and notation of crystals.

Example 4.1 $\left(T_{\Lambda}(\Lambda \in P)\right)$

Let $T_{\Lambda}=\left\{t_{\Lambda}\right\}$ with $\operatorname{wt}\left(t_{\Lambda}\right)=\Lambda, \varepsilon_{i}\left(t_{\Lambda}\right)=\varphi_{i}\left(t_{\Lambda}\right)=-\infty, \widetilde{e}_{i} t_{\Lambda}=\widetilde{f}_{i} t_{\Lambda}=0$. Tensoring a crystal $B$ with the crystal $T_{\Lambda}$ has the effect of shifting the weight $\mathrm{wt}$ by $\Lambda$ and leaving the other data fixed.

Example 4.2 $\left(B_{i}(i \in I)\right) B_{i}=\left\{b_{i}(n) ; n \in \mathbb{Z}\right\}$ with $\mathrm{wt}\left(b_{i}(n)\right)=n \alpha_{i}, \varepsilon_{i}\left(b_{i}(n)\right)=-n=-\varphi_{i}\left(b_{i}(n)\right)$, $\varepsilon_{j}\left(b_{i}(n)\right)=-\infty=\varphi_{j}\left(b_{i}(n)\right)$ if $j \neq i$; $\widetilde{e}_{i} b_{i}(n-1)=b_{i}(n)=\widetilde{f}_{i} b_{i}(n+1), \widetilde{e_{j}} b_{i}(n)=\widetilde{f}_{j} b_{i}(n)=0$ if $j \neq i$. We write $b_{i}$ for $b_{i}(0)$.

\subsection{Description of $B(\infty)$}

$B(\infty)$ is the crystal associated with the crystal graph of $\mathbf{U}_{q}^{-}(\mathfrak{g})$ where $\mathfrak{g}$ is the Kac-Moody algebra defined from the Cartan data above. One can also define $B(\infty)$ as an abstract crystal. As such, it can be characterized by Kashiwara-Saito's Proposition 4.3 below.

Proposition 4.3 ([KS97] Proposition 3.2.3) Let $B$ be a crystal and $b_{0}$ an element of $B$ with weight zero. Assume the following conditions.

(B1) $\operatorname{wt}(B) \subset Q_{-}$.

(B2) $b_{0}$ is the unique element of $B$ with weight zero.

(B3) $\varepsilon_{i}\left(b_{0}\right)=0$ for every $i \in I$.

(B4) $\varepsilon_{i}(b) \in \mathbb{Z}$ for any $b \in B$ and $i \in I$.

(B5) For every $i \in I$, there exists a strict embedding $\Psi_{i}: B \rightarrow B \otimes B_{i}$.

(B6) $\Psi_{i}(B) \subset B \times\left\{\tilde{f}_{i}^{n} b_{i} ; n \geq 0\right\}$.

(B7) For any $b \in B$ such that $b \neq b_{0}$, there exists $i$ such that $\Psi_{i}(b)=b^{\prime} \otimes \tilde{f}_{i}^{n} b_{i}$ with $n>0$.

Then $B$ is isomorphic to $B(\infty)$.

\section{Module theoretic realizations of certain crystals}

Let $\mathcal{B}$ denote the set of isomorphism classes of irreducible $R$-modules. Let $\mathbf{0}$ denote the zero module.

Let $M$ be an irreducible $R(\nu)$-module, so that $[M] \in \mathcal{B}$. By abuse of notation, we identify $M$ with $[M]$ in the following definitions. Hence, we are defining operators and functions on $\mathcal{B} \sqcup\{0\}$ below.

Recall from Section 3.2 the definitions of $\widetilde{e}_{i}, \widetilde{f}_{i}, \varepsilon_{i}$. For $\nu=\sum_{i \in I} \nu_{i} \alpha_{i}, i \in I$ and $M \in R(\nu)-\bmod$ set

$$
\operatorname{wt}(M)=-\nu, \quad \operatorname{wt}_{i}(M)=\left\langle h_{i}, \operatorname{wt}(M)\right\rangle, \quad \varphi_{i}(M)=\varepsilon_{i}(M)+\left\langle h_{i}, \operatorname{wt}(M)\right\rangle .
$$

Proposition 5.1 The tuple $\left(\mathcal{B}, \varepsilon_{i}, \varphi_{i}, \widetilde{e}_{i}, \widetilde{f}_{i}\right.$, wt) defines a crystal. 
We write $\mathbb{1} \in \mathcal{B}$ for the class of the trivial $R(\nu)$-module where $\nu=\emptyset$ and $|\nu|=0$.

One of the main theorems of this paper is Theorem 7.4 that identifies the crystal $\mathcal{B}$ as $B(\infty)$. However we need the many auxiliary results that follow before we can prove this.

Let $\mathcal{B}^{\Lambda}$ denote the set of isomorphism classes of irreducible $R^{\Lambda}$-modules. As in the previous section, by abuse of notation we write $\mathcal{M}$ for $[\mathcal{M}]$ below. Recall from Section 3.3 the definitions of $\widetilde{e}_{i}{ }^{\Lambda}, \widetilde{f}_{i}{ }^{\Lambda}, \varepsilon_{i}^{\Lambda}$. Let $\mathrm{wt}^{\Lambda}(\mathcal{M})=-\nu+\Lambda$ when $\mathcal{M}$ is an $R^{\Lambda}(\nu)$-module and $\varphi_{i}^{\Lambda}(\mathcal{M})=\max \left\{k \in \mathbb{Z} \mid \operatorname{pr}_{\Lambda} \circ \widetilde{f}_{i}^{k} \circ \operatorname{infl} \mathcal{M}_{\Lambda} \neq \mathbf{0}\right\}$. Note $\varepsilon_{i}^{\Lambda}(\mathcal{M})=\max \left\{k \in \mathbb{Z} \mid\left(\widetilde{e}_{i}^{\Lambda}\right)^{k} \mathcal{M} \neq \mathbf{0}\right\}$, and $0 \leq \varphi_{i}^{\Lambda}(\mathcal{M})<\infty$.

It is true, but not at all obvious, that with this definition $\varphi_{i}^{\Lambda}(\mathcal{M})=\varepsilon_{i}^{\Lambda}(\mathcal{M})+\left\langle h_{i}\right.$, wt $\left.{ }^{\Lambda} \mathcal{M}\right\rangle$; see Corollary 6.18 The proof that the data $\left(\mathcal{B}^{\Lambda}, \varepsilon_{i}^{\Lambda}, \varphi_{i}^{\Lambda},{\widetilde{e_{i}}}^{\Lambda}, \widetilde{f}_{i}{ }^{\Lambda}, \mathrm{wt}^{\Lambda}\right)$ defines a crystal is delayed until Section 7

\section{Understanding $R(\nu)$-modules and the crystal data of $\mathcal{B}$}

This section contains a summary of how the quantities $\varepsilon_{j}^{\vee}, \varepsilon_{i}, \varphi_{i}^{\Lambda}$ change with the application of $\widetilde{f}_{j}$.

Throughout this section we assume $j \neq i$ and set $a=a_{i j}=-\left\langle h_{i}, \alpha_{j}\right\rangle$.

\subsection{Jump}

Given an irreducible module $M, \operatorname{pr}_{\Lambda} \widetilde{f}_{i} M$ is either irreducible or zero. In the following subsection, we measure exactly when the latter occurs. More specifically, we compare $\varepsilon_{i}^{\vee}(M)$ to $\varepsilon_{i}^{\vee}\left(\widetilde{f}_{i} M\right)$ and compute when the latter "jumps" by +1 . In this case, we show $\widetilde{f}_{i} M \cong \widetilde{f}_{i}^{\vee} M$. Understanding exactly when this jump occurs is a key ingredient in constructing the strict embedding of crystals in Section 7.1.

Proposition 6.1 Let $M$ be an irreducible $R(\nu)$-module.

i) For any $i \in I$, either $\varepsilon_{i}^{\vee}\left(\tilde{f}_{i} M\right)=\varepsilon_{i}^{\vee}(M)$ or $\varepsilon_{i}^{\vee}(M)+1$.

ii) For any $i, j \in I$ with $i \neq j$, we have $\varepsilon_{i}^{\vee}\left(\tilde{f}_{j} M\right)=\varepsilon_{i}^{\vee}(M)$ and $\varepsilon_{i}\left(\tilde{f}_{j}^{\vee} M\right)=\varepsilon_{i}(M)$.

Definition 6.2 Let $M$ be an irreducible $R(\nu)$-module and let $\Lambda \in P^{+}$. Define $\varphi_{i}^{\Lambda}(M)=\max \{k \in \mathbb{Z} \mid$ $\left.\operatorname{pr}_{\Lambda} \widetilde{f}_{i}^{k} M \neq \mathbf{0}\right\}$, where we take the convention that $\widetilde{f}_{i}^{k}={\widetilde{e_{i}}}^{-k}$ when $k<0$.

Definition 6.3 Let $M$ be a simple $R(\nu)$-module and let $i \in I$. Then $\operatorname{jump}_{i}(M):=\max \{J \geq 0 \mid$ $\left.\varepsilon_{i}^{\vee}(M)=\varepsilon_{i}^{\vee}\left(\tilde{f}_{i}^{J} M\right)\right\}$.

Lemma 6.4 (Jump Lemma) Let $M$ be irreducible. The following are equivalent:

1) $\operatorname{jump}_{i}(M)=0$

3) $\widetilde{f}_{i}^{m} M \cong\left(\widetilde{f}_{i}^{\vee}\right)^{m} M$ for all $m \geq 1$

5) Ind $M \otimes L\left(i^{m}\right) \cong \operatorname{Ind} L\left(i^{m}\right) \otimes M$ for all $m \geq 1$

6) $\tilde{f}_{i} M \cong \operatorname{Ind} M \otimes L(i)$

7) Ind $M \otimes L(i)$ is irreducible

8) Ind $M \otimes L\left(i^{m}\right)$ is irreducible for all $m \geq 1$

9) $\varepsilon_{i}^{\vee}\left(\widetilde{f}_{i} M\right)=\varepsilon_{i}^{\vee}(M)+1$

10) $\operatorname{jump}_{i}\left(\widetilde{f}_{i}^{m} M\right)=0$ for all $m \geq 0$
2) $\tilde{f}_{i} M \cong \widetilde{f}_{i}^{\vee} M$

4) $\operatorname{Ind} M \otimes L(i) \cong$ Ind $L(i) \otimes M$

6') $\tilde{f}_{i}^{\vee} M \cong \operatorname{Ind} L(i) \otimes M$

$\left.7^{\prime}\right)$ Ind $L(i) \otimes M$ is irreducible

$\left.8^{\prime}\right)$ Ind $L\left(i^{m}\right) \otimes M$ is irreducible for all $m \geq 1$

9') $\varepsilon_{i}\left(\tilde{f}_{i}^{\vee} M\right)=\varepsilon_{i}(M)+1$

11) $\varepsilon_{i}^{\vee}\left(\widetilde{f}_{i}^{m} M\right)=\varepsilon_{i}^{\vee}(M)+m$ for all $m \geq 1$ 
Proposition 6.5 Let $M$ be a simple $R(\nu)$-module and let $i \in I$. Then the following hold.

i) $\operatorname{jump}_{i}(M)=\max \left\{J \geq 0 \mid \varepsilon_{i}(M)=\varepsilon_{i}\left(\left(\widetilde{f}_{i}^{\vee}\right)^{J} M\right)\right\}$

ii) $\operatorname{jump}_{i}(M)=\min \left\{J \geq 0 \mid \widetilde{f}_{i}\left(\widetilde{f}_{i}^{J} M\right) \cong \widetilde{f}_{i}^{\vee}\left(\widetilde{f}_{i}^{J} M\right)\right\}$

iii) $\operatorname{jump}_{i}(M)=\min \left\{J \geq 0 \mid \widetilde{f}_{i}\left(\left(\tilde{f}_{i}^{\vee}\right)^{J} M\right) \cong \widetilde{f}_{i}^{\vee}\left(\left(\widetilde{f}_{i}^{\vee}\right)^{J} M\right)\right\}$

iv) If $\varphi_{i}^{\Lambda}(M)>-\infty$, then $\operatorname{jump}_{i}(M)=\varphi_{i}^{\Lambda}(M)+\varepsilon_{i}^{\vee}(M)-\lambda_{i}$.

v) $\operatorname{jump}_{i}(M)=\varepsilon_{i}(M)+\varepsilon_{i}^{\vee}(M)+\mathrm{wt}_{i}(M)$.

Remark 6.6 Given $\Lambda, \Omega \in P^{+}$and irreducible modules $A$ and $B$ with $\operatorname{pr}_{\Lambda} A \neq \mathbf{0}, \operatorname{pr}_{\Omega} A \neq \mathbf{0}, \operatorname{pr}_{\Lambda} B \neq$ $\mathbf{0}, \operatorname{pr}_{\Omega} B \neq \mathbf{0}$, then $\varphi_{i}^{\Lambda}(A)-\varphi_{i}^{\Lambda}(B)=\varphi_{i}^{\Omega}(A)-\varphi_{i}^{\Omega}(B)$ since by Proposition 6.5 (iv) we compute

$$
\begin{aligned}
\varphi_{i}^{\Lambda}(A)-\varphi_{i}^{\Lambda}(B) & \left.=\operatorname{jump}_{i}(A)-\varepsilon_{i}^{\vee}(A)+\lambda_{i}\right)-\left(\operatorname{jump}_{i}(B)-\varepsilon_{i}^{\vee}(B)+\lambda_{i}\right) \\
& =\operatorname{jump}_{i}(A)-\operatorname{jump}_{i}(B)+\varepsilon_{i}^{\vee}(B)-\varepsilon_{i}^{\vee}(A)=\varphi_{i}^{\Omega}(A)-\varphi_{i}^{\Omega}(B) .
\end{aligned}
$$

\subsection{The Structure Theorems for simple $R(c i+j)$-modules}

In this section we describe the structure of all simple $R(c i+j)$-modules. We will henceforth refer to Theorems 6.7. 6.8 as the Structure Theorems for simple $R(c i+j)$-modules. Throughout this section we assume $j \neq i$ and set $a=a_{i j}=-\left\langle h_{i}, \alpha_{j}\right\rangle$.

In the theorems below we introduce the notation $\mathcal{L}\left(i^{c-n} j i^{n}\right)$ and $\mathcal{L}(n) \stackrel{\text { def }}{=} \mathcal{L}\left(i^{a-n} j i^{n}\right)$ for the simple $R(c i+j)$-modules (up to grading shift) when $c \leq a$. They are characterized by $\varepsilon_{i}\left(\mathcal{L}\left(i^{c-n} j i^{n}\right)\right)=n$.

Theorem 6.7 Let $c \leq a$ and let $\nu=c i+j$. Up to isomorphism and grading shift, there exists a unique irreducible $R(\nu)$-module denoted $\mathcal{L}\left(i^{c-n} j i^{n}\right)$ with $\varepsilon_{i}\left(\mathcal{L}\left(i^{c-n} j i^{n}\right)\right)=n$ for each $n$ with $0 \leq n \leq c$. Furthermore, $\varepsilon_{i}^{\vee}\left(\mathcal{L}\left(i^{c-n} j i^{n}\right)\right)=c-n$ and

$$
\operatorname{ch}\left(\mathcal{L}\left(i^{c-n} j i^{n}\right)\right)=[c-n]_{i} ![n]_{i} ! i^{c-n} j i^{n} .
$$

In particular, in the Grothendieck group $e_{i}^{(c-s)} e_{j} e_{i}^{(s)}\left[\mathcal{L}\left(i^{c-n} j i^{n}\right)\right]=0$ unless $s=n$.

In the previous theorem we introduced the notation $\mathcal{L}\left(i^{c-n} j i^{n}\right)$ for the unique (up to isomorphism and grading shift) simple $R(c i+j)$-module with $\varepsilon_{i}=n$ when $c \leq a$. Theorem 6.8 below extends this uniqueness to $c \geq a$. In the special case that $c=a$, we denote $\mathcal{L}(n)=\mathcal{L}\left(i^{a-n} j i^{n}\right)$. The following theorem motivates why we distinguish the special case $c=a$.

Theorem 6.8 Let $0 \leq n \leq a$.

i) The module

$$
\text { Ind } L\left(i^{m}\right) \otimes \mathcal{L}(n) \cong \operatorname{Ind} \mathcal{L}(n) \otimes L\left(i^{m}\right)
$$

is irreducible for all $m \geq 0$.

ii) Let $c \geq a$. Let $N$ be an irreducible $R(c i+j)$-module with $\varepsilon_{i}(N)=n$. Then $c-a \leq n \leq c$, and up to grading shift

$$
N \cong \operatorname{Ind} \mathcal{L}(n-(c-a)) \otimes L\left(i^{c-a}\right) .
$$

The proofs of the Structure Theorems are given by careful calculations on special weight bases of the above modules. In the interest of space, they are ommitted here. 


\subsection{Understanding $\varphi_{i}^{\Lambda}$}

The following theorems measure how the crystal data differs for $M$ and $\widetilde{f}_{j} M$.

Theorem 6.9 Let $M$ be a simple $R(\nu)$-module and let $\Lambda \in P^{+}$such that $\operatorname{pr}_{\Lambda} M \neq \mathbf{0}$ and $\operatorname{pr}_{\Lambda} \widetilde{f}_{j} M \neq \mathbf{0}$. Let $m=\varepsilon_{i}(M), k=\varphi_{i}^{\Lambda}(M)$. Then there exists an $n$ with $0 \leq n \leq a$ such that $\varepsilon_{i}\left(\widetilde{f}_{j} M\right)=m-(a-n)$ and $\varphi_{i}^{\Lambda}\left(\tilde{f}_{j} M\right)=k+n$.

Proof: This follows from Theorem 6.16 which proves the theorem in the case $\nu=c i+d j$ and from Proposition 6.17 which reduces it to this case.

One important rephrasing of the Theorem is

$$
\varphi_{i}^{\Lambda}\left(\widetilde{f}_{j} M\right)-\varepsilon_{i}\left(\widetilde{f}_{j} M\right)=a+\left(\varphi_{i}^{\Lambda}(M)-\varepsilon_{i}(M)\right)=-\left\langle h_{i}, \alpha_{j}\right\rangle+\left(\varphi_{i}^{\Lambda}(M)-\varepsilon_{i}(M)\right) .
$$

First we introduce several lemmas that will be needed.

Lemma 6.10 Suppose $c+d \leq a$.

i) Ind $\mathcal{L}\left(i^{c} j i^{d}\right) \otimes L\left(i^{m}\right)$ has irreducible cosocle equal to

$$
\tilde{f}_{i}{ }^{m} \mathcal{L}\left(i^{c} j i^{d}\right)=\widetilde{f}_{i}{ }^{m+d} \mathcal{L}\left(i^{c} j\right)=\left\{\begin{array}{cc}
\operatorname{Ind} \mathcal{L}(a-c) \otimes L\left(i^{m-a+c+d}\right) & m \geq a-(c+d) \\
\mathcal{L}\left(i^{c} j i^{d+m}\right) & m<a-(c+d) .
\end{array}\right.
$$

ii) Suppose there is a nonzero map Ind $\mathcal{L}\left(c_{1}\right) \otimes \mathcal{L}\left(c_{2}\right) \otimes \cdots \otimes \mathcal{L}\left(c_{r}\right) \otimes L\left(i^{m}\right) \longrightarrow Q$ where $Q$ is irreducible. Then $\varepsilon_{i}(Q)=m+\sum_{t=1}^{r} c_{t}$ and $\varepsilon_{i}^{\vee}(Q)=m+\sum_{t=1}^{r}\left(a-c_{t}\right)$.

iii) Let $B$ and $Q$ be simple with a nonzero map $\operatorname{Ind} B \otimes \mathcal{L}(c) \rightarrow Q$. Then $\varepsilon_{i}(Q)=\varepsilon_{i}(B)+c$.

Lemma 6.11 Let $N$ be an irreducible $R(c i+d j)$-module with $\varepsilon_{i}(N)=0$. Suppose $c+d>0$.

i) There exists irreducible $\bar{N}$ with $\varepsilon_{i}(\bar{N})=0$ and a surjection $\operatorname{Ind} \bar{N} \otimes \mathcal{L}\left(i^{b} j\right) \rightarrow N$ with $b \leq a$.

ii) There exists an $r \in \mathbb{N}$ and $b_{t} \leq$ a for $1 \leq t \leq r$ such that $\operatorname{Ind} \mathcal{L}\left(i^{b_{1}} j\right) \otimes \mathcal{L}\left(i^{b_{2}} j\right) \otimes \cdots \otimes \mathcal{L}\left(i^{b_{r}} j\right) \rightarrow N$.

Lemma 6.12 Suppose $Q$ is irreducible and we have a surjection

$$
\text { Ind } \mathcal{L}\left(i^{b_{1}} j\right) \otimes \mathcal{L}\left(i^{b_{2}} j\right) \otimes \cdots \otimes \mathcal{L}\left(i^{b_{r}} j\right) \otimes L\left(i^{h}\right) \rightarrow Q .
$$

i) Then for $h \gg 0$ we have a surjection Ind $\mathcal{L}\left(a-b_{1}\right) \otimes \mathcal{L}\left(a-b_{2}\right) \otimes \cdots \otimes \mathcal{L}\left(a-b_{r}\right) \otimes L\left(i^{g}\right) \rightarrow Q$ where $g=h-\sum_{t=1}^{r}\left(a-b_{t}\right)$.

ii) In the case $h<a r-\sum_{t=1}^{r} b_{t}$, we have

$$
\text { Ind } \mathcal{L}\left(i^{b_{1}} j\right) \otimes \cdots \otimes \mathcal{L}\left(i^{b_{s-1}} j\right) \otimes \mathcal{L}\left(i^{b_{s}} j i^{g^{\prime}}\right) \otimes \mathcal{L}\left(a-b_{s+1}\right) \otimes \cdots \otimes \mathcal{L}\left(a-b_{r}\right) \rightarrow Q
$$

where $g^{\prime}=h-\sum_{t=s+1}^{r}\left(a-b_{t}\right)$ and $s$ is such that $\sum_{t=s+1}^{r}\left(a-b_{t}\right) \leq h<\sum_{t=s}^{r}\left(a-b_{t}\right)$.

Lemma 6.13 Let $M$ be an irreducible $R(\nu)$-module and suppose Ind $A \otimes B \otimes L\left(i^{h}\right) \rightarrow M$ is a nonzero map where $\varepsilon_{i}(A)=0$ and $B$ is irreducible. Then there exists a surjective map Ind $A \otimes \widetilde{f}_{i}^{h} B \rightarrow M$. 
The following two lemmas discuss ways of detecting when $\operatorname{pr}_{\Lambda}$ of an induced module is zero.

Lemma 6.14 Let $A$ be an irreducible $R(\nu)$-module with $\operatorname{pr}_{\Lambda} A \neq \mathbf{0}$ and $k=\varphi_{i}^{\Lambda}(A)$.

i) Let $U$ be an irreducible $R(\mu)$-module and let $t \geq 1$. Then $\operatorname{pr}_{\Lambda} \operatorname{Ind} A \otimes L\left(i^{k+t}\right) \otimes U=\mathbf{0}$.

ii) Let $B$ be irreducible with $\varepsilon_{i}^{\vee}(B)>k$. Then $\operatorname{pr}_{\Lambda} \operatorname{Ind} A \otimes B=0$. In particular, if $Q$ is any irreducible quotient of Ind $A \otimes B$, then $\operatorname{pr}_{\Lambda} Q=\mathbf{0}$.

Lemma 6.15 Let $A$ be an irreducible $R(\nu)$-module with $\operatorname{pr}_{\Lambda} A \neq \mathbf{0}$ and $k=\varphi_{i}^{\Lambda}(A)$. Further suppose $\varepsilon_{i}(A)=\varepsilon_{j}(A)=0$ and that $B$ is an irreducible $R(c i+d j)$-module with $\varepsilon_{i}^{\vee}(B) \leq k$. Let $Q$ be irreducible such that Ind $A \otimes B \rightarrow Q$ is nonzero. Then $\varepsilon_{i}^{\vee}(Q) \leq \lambda_{i}$. Further, if $\varepsilon_{j}^{\vee}(B) \leq \varphi_{j}^{\Lambda}(A)$ (or if $\lambda_{j} \gg 0$ ) then $\operatorname{pr}_{\Lambda} Q \neq \mathbf{0}$.

Theorem 6.16 Let $M$ be an irreducible $R(c i+d j)$-module and let $\Lambda \in P^{+}$be such that $\operatorname{pr}_{\Lambda} M \neq \mathbf{0}$ and $\operatorname{pr}_{\Lambda} \widetilde{f}_{j} M \neq \mathbf{0}$. Let $m=\varepsilon_{i}(M), k=\varphi_{i}^{\Lambda}(M)$. Then there exists an $n$ with $0 \leq n \leq a$ such that $\varepsilon_{i}\left(\widetilde{f}_{j} M\right)=m-(a-n)$ and $\varphi_{i}^{\Lambda}\left(\widetilde{f}_{j} M\right)=k+n$.

We have just shown in Theorem 6.16 that Theorem 6.9 holds for all $R(c i+d j)$-modules. Next we show that to deduce the theorem for $R(\nu)$-modules for arbitrary $\nu$ it suffices to know the result for $\nu=c i+d j$.

Proposition 6.17 Let $\Lambda \in P^{+}$and let $M$ be an irreducible $R(\nu)$-module such that $\operatorname{pr}_{\Lambda} M \neq \mathbf{0}$ and $\operatorname{pr}_{\Lambda} \widetilde{f}_{j} M \neq \mathbf{0}$. Suppose $\varepsilon_{i}(M)=m$ and $\varepsilon_{i}\left(\widetilde{f}_{j} M\right)=m-(a-n)$ for some $0 \leq n \leq a$. Then there exists $c$, $d$ and an irreducible $R(c i+d j)$-module $B$ such that $\varepsilon_{i}(B)=m, \varepsilon_{i}\left(\widetilde{f}_{j} B\right)=m-(a-n)$ and there exists $\Omega \in P^{+}$with $\operatorname{pr}_{\Omega}(B) \neq \mathbf{0}, \operatorname{pr}_{\Omega}\left(\widetilde{f}_{j} B\right) \neq \mathbf{0}, \operatorname{pr}_{\Omega}(M) \neq \mathbf{0}, \operatorname{pr}_{\Omega}\left(\widetilde{f}_{j} M\right) \neq \mathbf{0}$, and furthermore

$$
\varphi_{i}^{\Omega}\left(\widetilde{f}_{j} M\right)-\varphi_{i}^{\Omega}(M)=\varphi_{i}^{\Omega}\left(\widetilde{f}_{j} B\right)-\varphi_{i}^{\Omega}(B) .
$$

Note that by Remark $6.6 \varphi_{i}^{\Lambda}\left(\widetilde{f}_{j} M\right)-\varphi_{i}^{\Lambda}(M)=\varphi_{i}^{\Omega}\left(\widetilde{f}_{j} M\right)-\varphi_{i}^{\Omega}(M)$, so once we prove this proposition, it together with Theorem 6.16 proves Theorem 6.9.

Corollary 6.18 (Corollary of Theorem 6.9) Let $\Lambda=\sum_{i \in I} \lambda_{i} \Lambda_{i} \in P^{+}$and let $M$ an irreducible $R(\nu)$ module such that $\operatorname{pr}_{\Lambda} M \neq \mathbf{0}$. Then

$$
\varphi_{i}^{\Lambda}(M)=\lambda_{i}+\varepsilon_{i}(M)+\mathrm{wt}_{i}(M) .
$$

\section{Main Results}

Now that we have built up the machinery of Section 6 , we can prove the module theoretic crystal $\mathcal{B}$ is isomorphic to $B(\infty)$. Once we have completed this step, it is not much harder to show $\mathcal{B}^{\Lambda} \cong B(\Lambda)$.

\subsection{Constructing the strict embedding $\Psi$}

Proposition 7.1 Let $M$ be a simple $R(\nu)$-module, and write $c=\varepsilon_{i}^{\vee}(M)$.

i) Suppose $\varepsilon_{i}^{\vee}\left(\widetilde{f}_{i} M\right)=\varepsilon_{i}^{\vee}(M)+1$. Then $\widetilde{e}_{i}^{\vee} \widetilde{f}_{i} M \cong M$ up to grading shift.

ii) Suppose $\varepsilon_{i}^{\vee}\left(\widetilde{f}_{j} M\right)=\varepsilon_{i}^{\vee}(M)$ where $i$ and $j$ are not necessarily distinct. Then $\left(\widetilde{e}_{i}^{\vee}\right)^{c}\left(\widetilde{f}_{j} M\right) \cong$ $\widetilde{f}_{j}\left(\widetilde{e}_{i}^{\vee}{ }^{\vee} M\right)$ up to grading shift. 
Proposition 7.2 Let $M$ be an irreducible $R(\nu)$-module, and write $c=\varepsilon_{i}^{\vee}(M), \bar{M}=\left(\widetilde{e}_{i}^{\vee}\right)^{c}(M)$.

i) $\varepsilon_{i}(M)=\max \left\{\varepsilon_{i}(\bar{M}), c-\mathrm{wt}_{i}(\bar{M})\right\}$.

ii) Suppose $\varepsilon_{i}(M)>0$. Then

$$
\varepsilon_{i}^{\vee}\left(\widetilde{e_{i}} M\right)= \begin{cases}c & \text { if } \varepsilon_{i}(\bar{M}) \geq c-\mathrm{wt}_{i}(\bar{M}), \\ c-1 & \text { if } \varepsilon_{i}(\bar{M})<c-\mathrm{wt}_{i}(\bar{M}) .\end{cases}
$$

iii) Suppose $\varepsilon_{i}(M)>0$. Then

$$
\left(\widetilde{e_{i}}\right)^{\varepsilon_{i}^{\vee}\left(\widetilde{e_{i}} M\right)}\left(\widetilde{e_{i}} M\right)= \begin{cases}\widetilde{e_{i}}(\bar{M}) & \text { if } \varepsilon_{i}(\bar{M}) \geq c-\mathrm{wt}_{i}(\bar{M}), \\ \bar{M} & \text { if } \varepsilon_{i}(\bar{M})<c-\mathrm{wt}_{i}(\bar{M}) .\end{cases}
$$

Proposition 7.3 For each $i \in I$ define a map $\Psi_{i}: \mathcal{B} \rightarrow \mathcal{B} \otimes B_{i}$ by

$$
M \mapsto\left(\widetilde{e}_{i}^{\vee}\right)^{c}(M) \otimes b_{i}(-c),
$$

where $c=\varepsilon_{i}^{\vee}(M)$. Then $\Psi_{i}$ is a strict embedding of crystals.

Theorem 7.4 (First Main Theorem) The crystal $\mathcal{B}$ is isomorphic to $B(\infty)$.

Now we will show the data $\left(\mathcal{B}^{\Lambda}, \varepsilon_{i}^{\Lambda}, \varphi_{i}^{\Lambda},{\widetilde{e_{i}}}^{\Lambda},{\widetilde{e_{i}}}^{\Lambda}\right.$, wt $\left.{ }^{\Lambda}\right)$ of Section 5 defines a crystal graph and identify it as the highest weight crystal $B(\Lambda)$.

Theorem 7.5 (Second Main Theorem) $\mathcal{B}^{\Lambda}$ is a crystal; the crystal $\mathcal{B}^{\Lambda}$ is isomorphic to $B(\Lambda)$.

\section{2 $\mathrm{U}_{q}^{+}$-module structures}

Set

$$
G_{0}^{*}(R)=\bigoplus_{\nu} G_{0}(R(\nu))^{*} \quad G_{0}^{*}\left(R^{\Lambda}\right)=\bigoplus_{\nu} G_{0}\left(R^{\Lambda}(\nu)\right)^{*}
$$

where, by $V^{*}$ we mean the linear dual $\operatorname{Hom}_{\mathcal{A}}(V, \mathcal{A})$. Because $G_{0}(R)$ and $G_{0}\left(R^{\Lambda}\right)$ are ${ }_{\mathcal{A}} \mathbf{U}_{q}^{+}$-modules, we can endow $G_{0}^{*}(R), G_{0}^{*}\left(R^{\Lambda}\right)$ with a left ${ }_{\mathcal{A}} \mathbf{U}_{q}^{+}$-module structure. $G_{0}(R(\nu))^{*}$ has basis given by $\left\{\delta_{M} \mid M \in \mathcal{B}, \operatorname{wt}(M)=-\nu\right\}$ defined by

$$
\delta_{M}([N])= \begin{cases}q^{-r} & M \cong N\{r\} \\ 0 & \text { otherwise }\end{cases}
$$

where $N$ ranges over simple $R(\nu)$-modules. We set $\operatorname{wt}\left(\delta_{M}\right)=-\operatorname{wt}(M)$. Likewise $G_{0}\left(R^{\Lambda}(\nu)\right)^{*}$ has basis $\left\{\mathfrak{d}_{\mathcal{M}} \mid \mathcal{M} \in \mathcal{B}^{\Lambda}, \operatorname{wt}(\mathcal{M})=-\nu+\Lambda\right\}$ defined similarly. Note that if $\delta_{M}$ has degree $d$ then $\delta_{M\{1\}}=q^{-1} \delta_{M}$ has degree $d-1$. Recall $\mathbb{1} \in \mathcal{B}$ denotes the trivial $R(0)$-module and we will also write $\mathbb{1} \in \mathcal{B}^{\Lambda}$ for the trivial $R^{\Lambda}(0)$-module.

Lemma 7.6 The maps $F:{ }_{\mathcal{A}} \mathbf{U}_{q}^{+} \rightarrow G_{0}^{*}(R), \quad \mathcal{F}:{ }_{\mathcal{A}} \mathbf{U}_{q}^{+} \rightarrow G_{0}^{*}\left(R^{\Lambda}\right)$ defined by $F(y)=y \cdot \delta_{\mathbb{1}}, \mathcal{F}(y)=$ $y \cdot \mathfrak{d}_{\mathbb{1}}$ respectively are surjective ${ }_{\mathcal{A}} \mathbf{U}_{q}^{+}$-module homomorphisms, and $\operatorname{ker} \mathcal{F} \ni e_{i}^{\left(\lambda_{i}+1\right)}$ for all $i \in I$. 
Recall that as a left ${ }_{\mathcal{A}} \mathbf{U}_{q}^{+}$-module ${ }_{\mathcal{A}} V^{*}(\Lambda) \cong{ }_{\mathcal{A}} \mathbf{U}_{q}^{+} / \sum_{i \in I}{ }_{\mathcal{A}} \mathbf{U}_{q}^{+} \cdot e_{i}^{\left(\lambda_{i}+1\right)}$. So the map $\mathcal{F}$ factors through ${ }_{\mathcal{A}} V^{*}(\Lambda)$, and the resulting induced map to $G_{0}^{*}\left(R^{\Lambda}\right)$ must be injective as the ranks of their weight spaces coincide by Theorem 7.5

Theorem 7.7 As ${ }_{\mathcal{A}} \mathbf{U}_{q}^{+}$modules

$$
\text { 1. }{ }_{\mathcal{A}} \mathbf{U}_{q}^{+} \cong G_{0}^{*}(R), \quad 2 \cdot{ }_{\mathcal{A}} V^{*}(\Lambda) \cong G_{0}^{*}\left(R^{\Lambda}\right), \quad 3 \cdot{ }_{\mathcal{A}} V(\Lambda) \cong G_{0}\left(R^{\Lambda}\right) .
$$

\section{References}

[Ari02] S. Ariki. Representations of quantum algebras and combinatorics of Young tableaux, volume 26 of University Lecture Series. AMS, Providence, RI, 2002.

[AK94] S. Ariki and K. Koike. A Hecke algebra of $(\mathbf{Z} / r \mathbf{Z})$ ? $\mathcal{S}_{n}$ and construction of its irreducible representations. Adv. Math., 106(2):216-243, 1994.

[BK09] J. Brundan and A. Kleshchev. Graded decomposition numbers for cyclotomic Hecke algebras. Adv. Math., 222(6):1883-1942, 2009.

[CR08] J. Chuang and R. Rouquier. Derived equivalences for symmetric groups and sl_2categorification. Ann. of Math., 167:245-298, 2008.

[KS97] M. Kashiwara and Y. Saito. Geometric construction of crystal bases. Duke Math. J., 89(1):936, 1997.

[KL09] M. Khovanov and A. Lauda. A diagrammatic approach to categorification of quantum groups I. Represent. Theory, 13:309-347, 2009.

[KL08a] M. Khovanov and A. Lauda. A diagrammatic approach to categorification of quantum groups II. To appear in Transactions of the AMS, math.QA/0804.2080, 2008.

[KL08b] M. Khovanov and A. Lauda. A diagrammatic approach to categorification of quantum groups III. math.QA/0807.3250, 2008.

[Lau08] A. D. Lauda. A categorification of quantum sl(2). math.QA/0803.3652, 2008.

[Lau09] A. D. Lauda. Nilpotency in type A cyclotomic quotients. math.RT/0903.2992, 2009.

[LV09] A. D. Lauda and M. Vazirani. Crystals from categorified quantum groups math.RT/0909.1810, 2009.

[Rou08] R. Rouquier. 2-Kac-Moody algebras. arXiv:0812.5023, 2008. 